\title{
Chemokine expression in tumor-to-tumor metastasis
}

\author{
JULIO S.N. PEGUERO ${ }^{1,3}$, LUIS H. CAMACHO ${ }^{1}$, BONNIE KEMP ${ }^{2}$ and LUIS T. CAMPOS ${ }^{1}$ \\ ${ }^{1}$ Department of Research, Oncology Consultants, Houston; ${ }^{2}$ Department of Pathology, Memorial Hermann, \\ Memorial City Medical Center, Houston, TX; ${ }^{3}$ University Medical Center at Brackenridge, \\ Department of Internal Medicine, University of Texas Medical Branch, Austin Programs, Austin, TX, USA
}

Received November 16, 2009; Accepted January 26, 2010

DOI: 10.3892/ol_00000079

\begin{abstract}
Chemokines play an important role in cancer metastasis by modulating the directional cell movement and migration of tumor cells. The most commonly overexpressed chemokine receptor in human cancer is CXCR4. Once activated by its ligand CXCL12 (stromal cell-derived factor-1 ligand/SDF1), CXCR4 stimulates several key migratory, proliferative and survival signaling cellular pathways. CXCR4 is expressed in small-cell lung carcinoma (SCLC) cells and other tumors. To further characterize the role of chemokines in tumor-to-tumor metastasis, we analyzed the tissue expression of CXCR4 and CXCL12 in the surgical specimen of a patient with this phenomenon. We performed immunohistochemical analysis for the expression of CXCR4 and CXCL12 in metastatic tumor tissue of a 69-year-old Caucasian male with extensive SCLC metastatic to a renal oncocytoma. The oncocytoma tissue harboring SCLC showed CXCL12 expression, but not CXCR4. A high expression of the two molecules was found in a normal renal parenchymal control. Our results suggest that CXCR4 and CXCL12 plays a role in this condition, but their expression may be affected by the microenvironment of the harboring malignancy. Further characterization of these phenomena is needed to shed light on the biological mechanisms of tumor metastasis.
\end{abstract}

\section{Introduction}

Chemokines were originally discovered and studied from the perspective of inflammation. However, their role in modulating directional cell movement and the migration of cancer cells has been demonstrated and is considered critical $(1,2)$.

The human chemokine system includes almost 50 chemokines and 14 receptors (2). The most commonly overexpressed chemokine in human cancer is CXCR4 (stromal cell-derived factor-1 receptor; fusin) and its ligrand CXCL12 (stromal cell-derived factor-1 ligand; SDF1 $\alpha$ ). CXCR4 activation by

Correspondence to: Dr Julio S.N. Peguero, Oncology Consultants P.A., 925 Gessner, Suite 600, Houston, TX 77024, USA

E-mail: jupesn@yahoo.com

Key words: stromal cell-derived factor ligand $1 \alpha$, stromal cellderived factor ligand $1 \alpha$ receptor, small-cell lung carcinoma cells
CXCL12 stimulates several key migratory, proliferative and survival signaling cellular pathways (3).

We hypothesized that CXCR4 and CXCL12, avidly expressed by small-cell lung carcinoma (SCLC) cells and documented to play important roles in the pathophysiology of metastasis, play a prominent role in the dissemination of tumoral cells and their subsequent invasion to a second malignancy.

\section{Case report}

A 69-year-old Caucasian male with a history of 60 packs/ year tobacco consumption and coronary artery disease was admitted to the hospital with a history of malaise, a 2-month 20-pound weight loss and a left kidney mass. The serum sodium level was $133 \mathrm{meq} / \mathrm{l}$ and hemoglobin, $15.5 \mathrm{~g} / \mathrm{dl}$. Laparoscopic partial nephrectomy was performed. A CT scan of the thorax was performed on the 5th post-operative day. The scan showed a mass-like right lobe opacification, bilateral pleural effusions and extensive mediastinal and right hilar adenopathy. Three days after discharge the patient was readmitted with acute dyspnea and atrial flutter. Chest X-rays confirmed a large right pleural effusion. The serum sodium level of $128 \mathrm{meq} / \mathrm{l}$ was due to a syndrome of inappropriate anti-diuretic hormone secretion. A right thoracenthesis was performed, and approximately 21 of fluid was removed. A bronchoscopy with transbronchial biopsy showed tumor invasion within the right main stem bronchus and nests of small-cell carcinoma with immunohistochemical stains positive for pankeratin, synaptophysin, CD56 and TTF1.

The patient was discharged from the hospital on demeclocycline for syndrome of inappropriate anti-diuretic hormone secretion, and cytotoxic chemotherapy was commenced with a combination of carboplatin and etoposide.

\section{Materials and methods}

Morphologic and immunohistochemical analysis. A left partial nephrectomy specimen was obtained measuring $4.5 \times 3.7 \times 3.2 \mathrm{~cm}$. Sectioning of the tissue revealed a single tan, focally hemorrhagic, well-circumscribed tumor, $2 \times 2 \times 2$ $\mathrm{cm}$, confined within the renal parenchyma. A histological examination showed that this tumor mass was composed of a primary renal oncocytoma containing multiple small islands of small-cell carcinoma. 
These foci of metastatic small-cell carcinoma were confined to the oncocytoma. No metastatic small-cell carcinoma was identified in the renal parenchyma surrounding the oncocytoma.

The oncocytoma itself exhibited classic histological features. The tumor was composed of homogeneous cells with abundant acidophilic granular cytoplasm and central to eccentrically located round nuclei with even chromatin (Fig. 1A). These cells formed solid compact nests (alveolar pattern) and areas of variably sized tubules set in the background of a loose edematous or hyalinized stroma. Mitotic activity was not appreciable. Immunohistochemical stains showed positive staining for EMA and negative staining for vimentin and colloidal iron.

The foci of the small-cell carcinoma present within the oncocytoma also exhibited classic features. The small foci were composed of solid sheets of small cells with extremely scant cytoplasm and nuclei with finely granular chromatin, absent or inconspicuous nucleoli and prominent nuclear moulding. Mitotic activity was rapid (Fig. 1B). Immunohistochemical stains confirmed the diagnosis with the cells showing positive staining for AE1/AE3, chromogranin, synaptophysin, CD56 and TTF-1.

Diagnosis was consistent with tumor-to-tumor metastasis with a primary SCLC as the site of origin and a renal oncocytoma as the harboring malignancy.

\section{Chemokine analysis}

Processing of the surgical specimens. Formalin-fixed and paraffin-embedded slides of normal renal parenchyma and tumor tissues for each antibody were hydrated. Antigens were retrieved in citrate buffer for $20 \mathrm{~min}$ and cooled at room temperature.

The slides were blocked for peroxidase activity in $3 \%$ hydrogen peroxide $\left(\mathrm{H}_{2} \mathrm{O}_{2}\right)$ for $5 \mathrm{~min}$ and rinsed in Tris buffer. The slides were also blocked for possible non-specific background staining with casein in PBS for $15 \mathrm{~min}$.

Staining for CXCL12 (SDF1 $\alpha$, mAB clone 7801B, IG1 class; R\&D Systems, Minneapolis, MN) and CXCR4 (fusin, goat polyclonal $\mathrm{AB}$, directed to the amino terminus of SDF1 $\alpha$ sc-6729; Santa Cruz Biotechnology, Santa Cruz, CA) was carried out at room temperature for 30 min using a 1:50 dilution. Slides were rinsed in Tris buffer.

For CXCL12, the secondary antibody, an anti-mouse IgG-HRP labeled polymer was applied for $30 \mathrm{~min}$. For CXCR4, an anti-goat IgG-HRP labeled polymer was applied for $10 \mathrm{~min}$. Slides were rinsed in Tris buffer twice. The chromogen, DAB, was applied for $5 \mathrm{~min}$ at room temperature and slides were rinsed in di $\mathrm{H}_{2} \mathrm{O}$ and counterstained in hematoxylin.

\section{Results}

CXCL12 antibody staining of the renal specimen showed an avid expression on the endothelial cell lining of vascular channels, glomeruli and tubules within the histological sections of the patient's oncocytoma and kidney parenchyma (Fig. 1C-F). CXCL12 also stained the SCLC cells within the oncocytoma (Fig. 1C-F). We then explored the CXCL12 and CXCR4 expression in a normal kidney control and confirmed their expression as well (data not shown). CXCR4 staining was not noted in the oncocytoma specimen nor were the small-cell carcinoma cells nested within the tumor.

\section{Discussion}

Several hypotheses have been proposed to explain the biology of tumor-to-tumor metastasis. In 1889, Paget in his 'seed and soil theory' mentioned that gross tumor development is a consequence of the provision of a fertile environment (the soil), in which compatible tumor cells (the seeds) proliferate. A mechanical theory was proposed by Ewing in 1928, describing site specificity as a direct consequence of the anatomical location of a primary tumor (4). High lipid and glycogen content in the kidney were also proposed as a potential mechanism to attract metastatic cells $(4,5)$. However, few malignancies metastasize to the kidneys.

More recent evidence links chemokines with the pathogenesis of metastasis in more than 23 human cancer cells including SCLC (6).

In vivo data showed that certain chemokines serve as tissue-specific attractant molecules for tumor cells, promoting tumor cell migration to a particular site through direct action of the chemokine ligands on chemokine receptors via the activation of heterotrimeric $G$ proteins. The $G$ protein subunits then stimulate multiple signal transduction pathways, involving the phosphatidylinositide 3 kinase (PIK-3)/Akt pathway and various Src family kinases (6).

SCLC cells use the CXCR4 receptor to migrate to the bone marrow tumor microenvironment, which is rich in CXCL12. This migration is due to the activation of integrins after CXCR4/CXCL12 involvement, allowing the cells to interact with extracellular matrix components (7).

The CXCR4/CXCL12 axis has been found to be up-regulated in renal cell carcinoma and other malignancies such as chronic lymphocytic leukemia, breast carcinoma, multiple myeloma, melanoma and ovarian carcinoma and may constitute a novel therapeutic target $(1,7)$.

The development of tumor metastasis from a second primary malignancy is uncommon and remains biologically puzzling. Its low incidence has made its full biological characterization evasive.

Initially described by Campbell, the event must meet the following criteria to be considered tumor-to-tumor metastasis: i) the presence of more than one primary tumor; ii) the recipient tumor has to be a true neoplasm; iii) there must be evidence of true metastasis from the second neoplasm; iv) the second malignancy must grow or invade the tissues of the hosting tumor; v) the metastatic growth must not be due to contiguous growth or embolism of tumor cells, and vi) tumors that metastasize to the lymphatic system where a lymphatic malignancy already exists are not considered tumor-to-tumor metastasis (4,8-10).

Renal cell carcinoma and meningioma are the most common malignant and benign recipients, respectively, whereas the lung is the most common metastatic donor in both settings (10).

Though inconclusive, our results suggest that the high tissue expression of CXCL12 observed in the tumor tissues is also present in normal kidney parenchyma (control sample). Contrary to our hypothesis, CXCR4 expression was not noted 


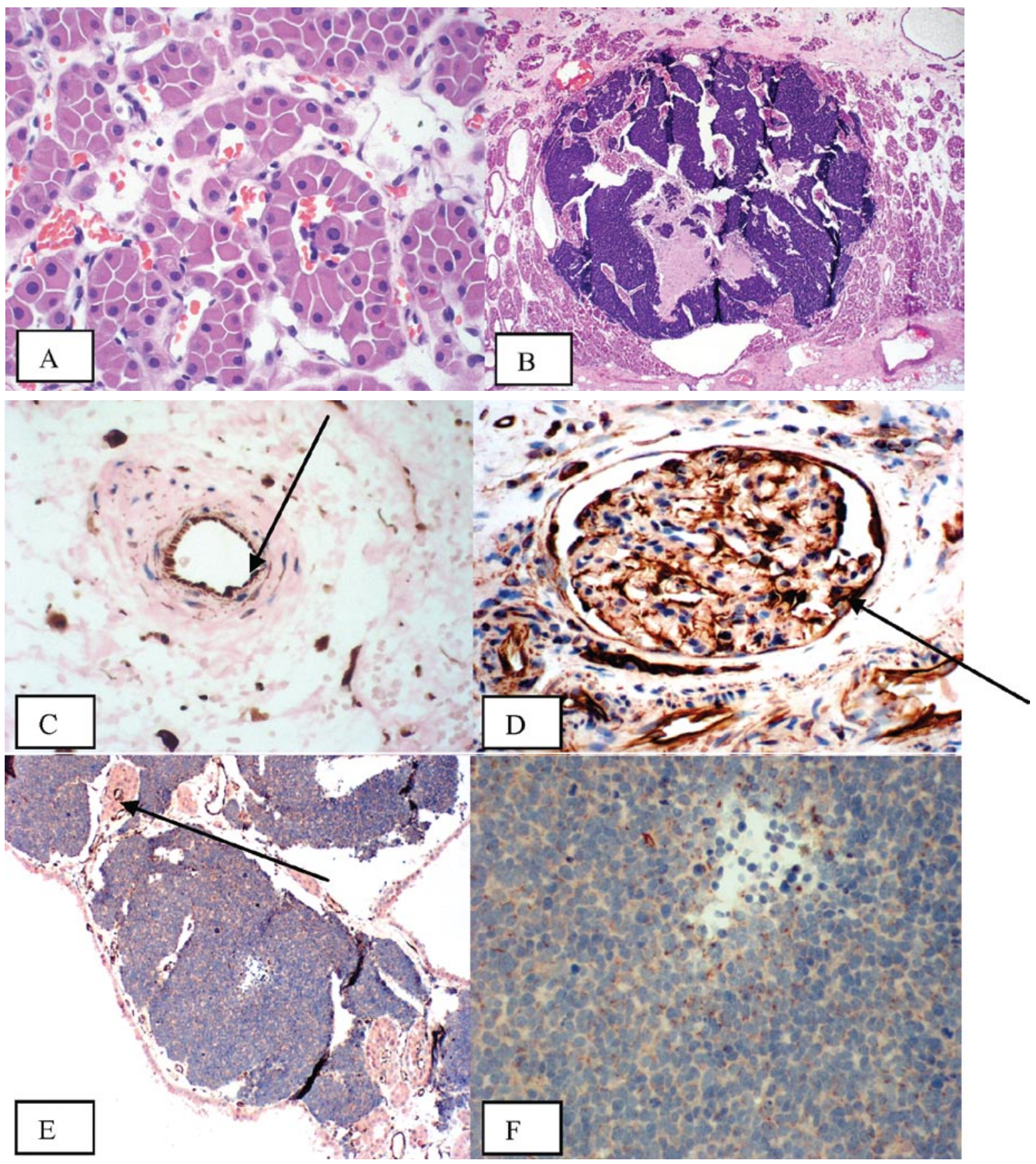

Figure 1. (A) Higher power field H\&E staining of the oncocytoma showing homogeneous cells with abundant acidophilic granular cytoplasm and central to eccentrically located round nuclei. (B) Lower power field of small-cell carcinoma nests present within the oncocytoma. (C) CXCL12 staining in the oncocytoma showing vascular channels and (D) glomeruli. CXCL12 staining in small-cell carcinoma cells within the oncocytoma; (E) lower power field and (F) higher power field.

in the SCLC metastasis in our case specimen. However, the lack of expression is perhaps related to the interaction of the oncocytoma's stromal microenvironment and the metastatic nests of SCLC cells. In this particular event the host for SCLC was indeed a renal neoplasm. Unlike oncocytomas, renal cell carcinoma cells induce CXCR4 transcription via hypoxia inducible factor (HIF1). Moreover, the up-regulation of prolyl and asparagynil hydroxylase have been identified in oncocytomas (11). These enzymes decrease the production of HIF1- $\alpha$ via proteasome degradation by down-regulating the transcription of CXCR4 $(11,12)$. This partially explains the absence of CXCR4 receptors in our specimen and likely plays a role in the low metastatic potential that oncocytomas exhibit.

The molecular events between CXCR4 and its ligand CXCL12 have yet to be elucidated in tumor-to-tumor metastasis. In this regard, the question remains as to whether the mechanism of the propagation of cells is related to the chemokine axis, the oncocytoma's microtumoral environment or, more likely, a combination of both. This preliminary 
observation warrants further investigation with functional molecular studies to characterize the environment surrounding chemokines in tumor-to-tumor metastasis.

\section{Acknowledgements}

The authors would like to thank Dr Jan Burger for the guidance and invaluable suggestions in interpretation of the chemokine staining.

\section{References}

1. Burger JA and Kipps TJ: CXCR4: a key receptor in the crosstalk between tumor cells and their microenvironment. Blood 5: 1761-1767, 2006.

2. Zlotnik A: Chemokines in neoplastic progression. Semin Cancer Biol 3: 181-185, 2004.

3. Hartmann TN, Burger JA, Glodek A, Fujii N and Burger M: CXCR4 chemokine receptor and integrin signaling co-operate in mediating adhesion and chemoresistance in small cell lung cancer (SCLC) cells. Oncogene 27: 4462-4471, 2005.

4. Campbell LV Jr, Gilbert E, Chamberlain CR Jr and Watne AL: Metastases of cancer to cancer. Cancer 3: 635-643, 1968.
5. Hart IR: 'Seed and soil' revisited: mechanisms of site-specific metastasis. Cancer Metastasis Rev 1: 5-16, 1982.

6. Tanaka T, Bai Z, Srinoulprasert Y, Yang BG, Hayasaka H and Miyasaka M: Chemokines in tumor progression and metastasis. Cancer Sci 6: 317-322, 2005.

7. Balkwill F: Cancer and the chemokine network. Nat Rev Cancer 7: 540-550, 2004.

8. Singh EO, Benson RC Jr and Wold LE: Cancer-to-cancer metastasis. J Urol 2: 340-342, 1984.

9. Ben-Izhak O and Lichtig C: Renal oncocytoma harbouring metastatic lung carcinoma. Case report. Scand J Urol Nephrol 4: 317-318, 1990.

10. Altinoz MA, Santaguida C, Guiot MC and Del Maestro RF: Spinal hemangioblastoma containing metastatic renal cell carcinoma in von Hippel-Lindau disease. Case report and review of the literature. J Neurosurg Spine 6: 495-500, 2005.

11. Koeman JM, Russell RC, Tan MH, Petillo D, Westphal M and Koelzer K: Somatic pairing of chromosome 19 in renal oncocytoma is associated with deregulated EGLN2-mediated [corrected] oxygen-sensing response. PLoS Genet 9: e1000176, 2008.

12. Ginouves A, Ilc K, Macias N, Pouyssegur J and Berra E: PHDs overactivation during chronic hypoxia 'desensitizes' HIFalpha and protects cells from necrosis. Proc Natl Acad Sci USA 12: 4745-4750, 2008. 